\title{
Inflammation and endothelial activation in early adulthood are associated with future emphysema: the CARDIA Lung Study
}

\author{
To the Editor:
}

Chronic obstructive pulmonary disease (COPD) is a heterogeneous syndrome that is characterised by inflammation, airflow limitation and emphysema [1]. Systemic inflammation is associated with decreased lung function and an accelerated decline in lung function over time [2-4], and it has been postulated that chronic inflammation accelerates pulmonary structural damage. In addition to systemic inflammation, differences in cell adhesion molecules, which are markers of endothelial activation, have been tied to accelerated progression of emphysema on computed tomography (CT) in a large community population [5]. However, whether endothelial activation or systemic inflammation predicts the development of emphysema remains unclear. We hypothesised that elevated circulating levels of high-sensitivity C-reactive protein (hs-CRP), fibrinogen, and the cellular adhesion molecules intercellular adhesion molecule (ICAM)-1 and P-selectin, measured in early adulthood, are associated with emphysema on CT in middle age in a general population-based sample.

The Coronary Artery Risk Development in Young Adults (CARDIA) study is a longitudinal cohort study of 5115 participants conducted in four US cities that has completed a series of examinations including year 0 (baseline; 1985-1986), year 7 and year 25 [6]. Participants were generally healthy with no evidence of chronic medical diseases at the time of enrolment. At year 25, 3498 participants attended the clinical visit (one withdrew consent; 1616 did not attend the year 25 visit). Participants were excluded from this analysis if they did not have a year 25 lung CT measure for visual emphysema $(n=329)$, did not attend the year 7 examination $(n=309)$, were missing data for a year 7 covariate $(n=66)$, or were missing data on all four measures of hs-CRP, fibrinogen, ICAM-1 and P-selectin $(n=54)$. Fibrinogen and hs-CRP were measured by nephelometry [3]; ICAM-1 and P-selectin were measured by ELISA [4] at year 7. Endothelial and inflammatory markers were categorised in quartiles. Visual assessment for emphysema on year $25 \mathrm{CT}$ scans was performed as previously described [7]. Briefly, a reader reviewed the scans and classified them as having centrilobular and/or paraseptal emphysema versus no emphysema, and a second expert reviewed all CT scans that had visualised emphysema as well as a random sample of $10 \%$ of the remaining CT scans. The kappa coefficient for centrilobular emphysema was 0.74 and was 0.70 for paraseptal emphysema. Cases in which the two readers disagreed were adjudicated by a third reader. Each reader was blinded to the categorisations performed by the other two.

Multivariable logistic regression was performed to evaluate the association between inflammatory and endothelial markers and evidence of emphysema identified on chest CT at year 25, adjusting for age, race/ sex, smoking status at year 7, pack-year history (year 7), body mass index (BMI) and field centre. We also performed analysis restricted to current smokers, who were most likely to develop emphysema. All analyses were performed in SAS (version 9.4; SAS Institute, Cary, NC, USA).

There were 2740 participants included in the analysis. As shown in table 1, participants were mean $\pm \mathrm{SD}$ $32.2 \pm 3.6$ years old, $45 \%$ male, $55 \%$ white, $45 \%$ black and had a BMI of $26.8 \pm 5.9 \mathrm{~kg} \cdot \mathrm{m}^{-2}$. The majority (59\%) were never smokers, $25 \%$ were current smokers and $16 \%$ were former smokers with a

@ERSpublications

Circulating markers of inflammation and endothelial activation predict future emphysema in a sample of generally healthy young adults http://ow.ly/7DCW30mqyvO

Cite this article as: Wells JM, Colangelo LA, Sivarajan L, et al. Inflammation and endothelial activation in early adulthood are associated with future emphysema: the CARDIA Lung Study. Eur Respir J 2019; 53: 1801532 [https://doi.org/10.1183/13993003.01532-2018]. 


\begin{tabular}{|c|c|c|c|c|}
\hline & Total cohort & Emphysema & No emphysema & p-value \\
\hline Participants & 2740 & 189 & 2551 & \\
\hline Age years & $32.2 \pm 3.6$ & $32.4 \pm 3.5$ & $32.2 \pm 3.6$ & 0.42 \\
\hline Race & & & & $<0.0001$ \\
\hline White & $1498(54.7 \%)$ & $67(35.4 \%)$ & $1431(56.1 \%)$ & \\
\hline Black & $1242(45.3 \%)$ & $122(64.6 \%)$ & $1120(43.9 \%)$ & \\
\hline Males & $1227(44.8 \%)$ & $112(59.3 \%)$ & $1115(43.7 \%)$ & $<0.0001$ \\
\hline Smoking status & & & & $<0.0001$ \\
\hline Current & $683(24.9 \%)$ & $154(81.5 \%)$ & $529(20.7 \%)$ & \\
\hline Former & $448(16.4 \%)$ & $12(6.4 \%)$ & $436(17.1 \%)$ & \\
\hline Never & $1609(58.7 \%)$ & $23(12.1 \%)$ & $1586(62.2 \%)$ & \\
\hline Smoking history at year 7 pack-years & $3.3 \pm 6.2$ & $10.4 \pm 8.6$ & $2.8 \pm 5.7$ & $<0.0001$ \\
\hline $\mathrm{BMI} \mathrm{kg} \cdot \mathrm{m}^{-2}$ & $26.8 \pm 5.9$ & $24.2 \pm 4.3$ & $27.0 \pm 6.0$ & $<0.0001$ \\
\hline Fibrinogen ${ }^{\#} \mathrm{mg} \cdot \mathrm{dL}^{-1}$ & $334.7 \pm 74.5$ & $340.7 \pm 81.3$ & $334.3 \pm 73.9$ & 0.26 \\
\hline $\mathrm{CRP}^{\mathbb{1}} \mu \mathrm{g} \cdot \mathrm{mL}^{-1}$ & $2.86 \pm 8.03$ & $3.75 \pm 7.34$ & $2.79 \pm 8.07$ & 0.12 \\
\hline P-selectin ${ }^{+} \mathrm{ng} \cdot \mathrm{mL}^{-1}$ & $22.23 \pm 7.82$ & $24.57 \pm 6.56$ & $22.06 \pm 7.88$ & $<0.0001$ \\
\hline ICAM-1 ${ }^{\S} \mathrm{ng} \cdot \mathrm{mL}^{-1}$ & $196.6 \pm 76.5$ & $254.2 \pm 110.8$ & $192.3 \pm 71.4$ & $<0.0001$ \\
\hline
\end{tabular}

Data are presented as mean $\pm S D$, unless otherwise stated. t-test and Chi-squared testing were used for bivariate analysis of continuous and categorical variables, respectively. BMI: body mass index; CRP: C-reactive protein; ICAM: intercellular adhesion molecule. " ${ }^{\#}: 27$ participants missing this measure; " 24 participants missing this measure; ${ }^{+}: 64$ participants missing this measure; ${ }^{\S}: 826$ participants missing this measure.

3.3 \pm 6.2 pack-year history. At year 7 , plasma fibrinogen was $334.7 \pm 74.5 \mathrm{mg} \cdot \mathrm{dL}^{-1}$, hs-CRP was 2.86 $\pm 8.03 \mu \mathrm{g} \cdot \mathrm{mL}^{-1}$, P-selectin was $22.23 \pm 7.82 \mathrm{ng} \cdot \mathrm{mL}^{-1}$ and ICAM-1 was $196.6 \pm 76.5 \mathrm{ng} \cdot \mathrm{mL}^{-1}$.

Emphysema was present in 189 (7\%) of participants at follow-up. Clinical factors associated with future emphysema included race, male sex, current smoking status and lower BMI. Associations between circulating markers of systemic inflammation and endothelial activation at year 7 and future emphysema at year 25 are shown in table 2. The highest quartile of hs-CRP (adjusted odds ratio (aOR) 2.31, 95\% CI 1.36-3.95) was associated with greater odds of emphysema as compared to the lowest quartile after adjustment for age, race/sex, centre, smoking status, pack-years and BMI. Likewise, the highest quartile of ICAM-1 (aOR 2.09, 95\% CI 1.16-3.78) was independently associated with incident emphysema after adjusting for the same covariates. In analyses restricted to current smokers, we observed similar associations between the highest quartiles of fibrinogen, hs-CRP, P-selectin and emphysema (table 2).

In this population-based cohort study of young adults, we report that markers of systemic inflammation as well as markers of endothelial activation are associated with emphysema detected on CT 18 years later. These associations were adjusted for traditional risk factors for COPD, including baseline cigarette smoking status, indicating these markers may serve as prognostic indicators and potential therapeutic targets for intervention prior to development of structural lung disease.

Previous studies evaluating the associations between the inflammatory markers hs-CRP and fibrinogen with emphysema have yielded conflicting results. In an analysis of COPDGene and ECLIPSE (Evaluation of COPD Longitudinally to Identify Predictive Surrogate Endpoints), fibrinogen and hs-CRP, when added to surfactant protein D and soluble receptor for advanced glycation end-products (sRAGE), were associated with quantitative emphysema severity and emphysema progression over time [8]. Conversely, cross-sectional analysis in the AERIS (Acute Exacerbation and Respiratory Infections in COPD) study found inverse associations between quantitative emphysema and fibrinogen and hs-CRP [9]. The current study extends our understanding of these findings to show that elevated hs-CRP and fibrinogen, markers of systemic inflammation, may serve as a risk factor for emphysema development.

It is likely that ICAM-1 and P-selectin may be involved in emphysema pathogenesis through their roles in leukocyte recruitment and facilitating inflammatory processes in the lung that lead to lung destruction. In the MESA (Multi-Ethnic Study of Atherosclerosis) Lung Study, ICAM-1, but not P-selectin, was associated with the progression of percentage emphysema measured serially over a 10-year time period [5]. In murine models, blockade of ICAM-1 prevents the development of experimental emphysema, strengthening the plausibility of its potential causal role in emphysema development [10]. 
TABLE 2 Association (covariate adjusted odds ratio ${ }^{\#}$ ) between year 7 inflammatory markers (mean age 32 years) and emphysema 18 years later (mean age 50 years)

\begin{tabular}{|c|c|c|}
\hline & \multicolumn{2}{|c|}{ Visual emphysema } \\
\hline & Total cohort & Current smokers \\
\hline \multicolumn{3}{|l|}{ Fibrinogen $\mathrm{mg} \cdot \mathrm{dL}^{-1}$} \\
\hline Q1: 107-281 & Ref. & Ref. \\
\hline Q2: 282-323 & $1.10(0.67-1.80)$ & $1.27(0.69-2.33)$ \\
\hline Q3: 324-374 & $1.02(0.62-1.68)$ & $1.51(0.84-2.71)$ \\
\hline Q4: 375-714 & $1.67(0.99-2.83)$ & $2.23 *(1.19-4.20)$ \\
\hline \multicolumn{3}{|l|}{ hs-CRP $\mu \mathrm{g} \cdot \mathrm{mL}^{-1}$} \\
\hline Q1: $0.15-0.48$ & Ref. & Ref. \\
\hline Q2: $0.49-1.08$ & $1.19(0.72-1.97)$ & $1.14(0.62-2.10)$ \\
\hline Q3: 1.09-3.03 & $1.32(0.78-2.21)$ & $1.51(0.83-2.78)$ \\
\hline Q4: $3.04-333.0$ & $2.31 *(1.36-3.95)$ & $2.30 *(1.22-4.35)$ \\
\hline \multicolumn{3}{|l|}{$\mathrm{P}$-selectin $\mathrm{ng} \cdot \mathrm{mL}^{-1}$} \\
\hline Q1: $2.98-16.81$ & Ref. & Ref. \\
\hline Q2: 16.82-21.49 & $1.51(0.86-2.66)$ & $1.82(0.88-3.76)$ \\
\hline Q3: 21.50-26.64 & $1.70(0.98-2.96)$ & $2.63 *(1.32-5.25)$ \\
\hline Q4: 26.65-103.70 & $1.65(0.94-2.88)$ & $2.29 *(1.14-4.61)$ \\
\hline \multicolumn{3}{|l|}{ ICAM-1 ng. $\mathrm{mL}^{-1}$} \\
\hline Q1: 0.9-151.9 & Ref. & Ref. \\
\hline Q2: $152.0-190.2$ & $1.38(0.71-2.68)$ & $0.94(0.41-2.17)$ \\
\hline Q3: 190.3-234.3 & 1.39 (0.72-2.69) & $1.00(0.44-2.24)$ \\
\hline Q4: $234.4-671.3$ & $2.09 *(1.16-3.78)$ & $1.69(0.87-3.30)$ \\
\hline \multicolumn{3}{|c|}{$\begin{array}{l}\text { Data are presented as adjusted odds ratio }(95 \% \mathrm{CI}) \text {. Q: quartile; hs-CRP: high-sensitivity C-reactive protein; } \\
\text { ICAM: intercellular adhesion molecule. \#: logistic regression models were adjusted for age, race/sex } \\
\text { group, smoking status, pack-years (at year } 7) \text {, body mass index and clinical centre; }{ }^{\text {: }} \text { : analyses limited to } \\
\text { current smokers at year } 7 .{ }^{*}: p<0.05 \text {. }\end{array}$} \\
\hline
\end{tabular}

The major strengths of our study are the recruitment and inclusion of healthy young adults, and the long duration of follow-up. These features allow us to investigate factors that may identify risk for development of disease in middle age or later in life. While our findings are observational, the measurement of these markers decades before evaluation for emphysema helps to establish temporality of our observations. Recapitulating the findings in current smokers at year 7 suggests these relationships are not simply the result of current smoking, but also may indicate individuals at highest risk for future emphysema. Additionally, while these markers were selected based on biological plausibility, it is likely that other markers of inflammation such as surfactant protein D or sRAGE (among others) may also identify this systemic inflammatory endotype that is at risk for emphysema development.

Our study has several limitations. First, the CARDIA scans were acquired with cardiac gating. The image acquisition starts and stops based upon synchronisation with the cardiac cycle. This gating leads to a motion artefact on the axial slices, which have lower density than the surrounding parenchyma and can be incorrectly labelled as "emphysema". Those CARDIA participants that have emphysema generally have mild or very mild changes in their parenchyma. We applied objective methods to the CARDIA CT scans and found that this gating artefact added significant noise to our estimates of disease and effectively offset the added sensitivity for disease detection one observes in non-gated CT scans. Visual inspection is not confounded by such gating artefact and for this reason we chose to use subjective assessments of the parenchyma as our outcome of interests. Second, the cohort was composed primarily of healthy young adults without comorbidities so we did not adjust findings based on comorbid conditions. Finally, only a small proportion of the individuals studied developed emphysema, potentially influencing our findings.

In conclusion, in this sample of generally healthy young adults, circulating markers of inflammation and endothelial activation predict future emphysema. Individuals with the highest levels of these markers had the greatest risk for developing emphysema in middle age, even when factors including smoking status were taken into consideration. These findings suggest that these markers could identify younger individuals most vulnerable to development of lung disease and may serve as therapeutic targets in preserving lung health.

J. Michael Wells $s^{1,2,3}$, Laura A. Colangelo ${ }^{4}$, Lakshmi Sivarajan ${ }^{5}$, Bharat Thyagarajan ${ }^{6}$, Mark T. Dransfield ${ }^{1,2,3}$, Carlos Iribarren ${ }^{7}$, Paul A. Reyfman ${ }^{4}$, David R. Jacobs Jr $\oplus^{8}$, George R. Washko9 and Ravi Kalhan $\oplus^{4,5}$ 
${ }^{1}$ Division of Pulmonary, Allergy, and Critical Care Medicine, Dept of Medicine, University of Alabama at Birmingham (UAB), Birmingham, AL, USA. ${ }^{2}$ UAB Lung Health Center, Birmingham, AL, USA. ${ }^{3}$ Birmingham VA Medical Center, Birmingham, AL, USA. ${ }^{4}$ Dept of Preventive Medicine, Northwestern University Feinberg School of Medicine, Chicago, IL, USA. ${ }^{5}$ Asthma and COPD Program, Division of Pulmonary and Critical Care Medicine, Dept of Medicine, Northwestern University Feinberg School of Medicine, Chicago, IL, USA. ${ }^{6}$ Dept of Laboratory Medicine and Pathology, University of Minnesota, Minneapolis, MN, USA. ${ }^{7}$ Division of Research, Kaiser Permanente, Oakland, CA, USA. ${ }^{8}$ Division of Epidemiology and Community Health, School of Public Health, University of Minnesota, Minneapolis, MN, USA. ${ }^{9}$ Division of Pulmonary and Critical Care Medicine, Dept of Medicine, Brigham and Women's Hospital, Boston, MA, USA.

Correspondence: J. Michael Wells, Division of Pulmonary, Allergy, and Critical Care Medicine, Dept of Medicine, UAB, 1900 University Blvd, THT 422, Birmingham, AL 35294, USA. E-mail: jmwells@uabmc.edu

Received: Aug 132018 | Accepted after revision: Oct 172018

Conflict of interest: J.M. Wells reports receiving grants from the NIH/NHLBI during the conduct of the study; contracts to conduct research and advisory board membership for GlaxoSmithKline, contracts to conduct research for AstraZeneca, Bayer and Gilead, advisory board membership for Mereo BioPharma and Boehringer Ingelheim, and a research pilot project for the Cystic Fibrosis Foundation, outside the submitted work. L.A. Colangelo reports grants from NHLBI to Northwestern University during the conduct of the study. L. Sivarajan has nothing to disclose. B. Thyagarajan reports receiving grants from the National Institutes of Health during the conduct of the study. M.T. Dransfield reports receiving grants from the NIH during the conduct of the study; grants from the Dept of Defense, personal fees for consulting and fees for contracted clinical trials from Boehringer Ingelheim, GlaxoSmithKline, AstraZeneca, PneumRx/BTG and Boston Scientific, fees for contracted clinical trials from Novartis, Yungjin and Pulmonx, and personal fees for consulting from Genentech and Quark Pharmaceuticals, outside the submitted work. C. Iribarren has nothing to disclose. P.A. Reyfman has nothing to disclose. D.R. Jacobs has nothing to disclose. G.R. Washko reports receiving grants from the NIH; grants, and consultancy and advisory board fees from Boehringer Ingelheim; consultancy fees from Genentech, Regeneron and GlaxoSmithKline; fees for consultancy and chairing a data safety monitoring board from PulmonX; advisory fees from ModoSpira; grants from BTG Interventional Medicine; grants and consultancy fees from Janssen Pharmaceuticals; and advisory board fees from Toshiba, all outside the submitted work. G.R. Washko is a founder and co-owner of Quantitative Imaging Solutions, which provides image-based consulting and develops software to enable data sharing. G.R. Washko's spouse works for Biogen, which is focused on developing therapies for fibrotic lung disease. R. Kalhan reports receiving grants from the NHLBI during the conduct of the study; grants and personal fees from Boehringer Ingelheim, AstraZeneca and GlaxoSmithKline, grants from PneumRx (BTG) and Spiration, personal fees from CVS Caremark, Aptus Health and Boston Scientific, outside the submitted work.

Support statement: CARDIA is conducted and supported by the National Heart, Lung, and Blood Institute (NHLBI) in collaboration with the University of Alabama at Birmingham (grants HHSN268201800005I and HHSN268201800007I), Northwestern University (HHSN268201800003I), University of Minnesota (HHSN268201800006I) and Kaiser Foundation Research Institute (HHSN268201800004I). Additional funding was provided by NHLBI R01 HL122477 (CARDIA Lung Study) and K08 HL123940 (to J.M. Wells). This manuscript has been reviewed by CARDIA for scientific content. Funding information for this article has been deposited with the Crossref Funder Registry.

\section{References}

1 Vogelmeier CF, Criner GJ, Martinez FJ, et al. Global Strategy for the Diagnosis, Management, and Prevention of Chronic Obstructive Lung Disease 2017 Report: GOLD Executive Summary. Eur Respir J 2017; 49: 1700214.

2 Hancox RJ, Poulton R, Greene JM, et al. Systemic inflammation and lung function in young adults. Thorax 2007; 62: $1064-1068$

3 Kalhan R, Tran BT, Colangelo LA, et al. Systemic inflammation in young adults is associated with abnormal lung function in middle age. PLoS One 2010; 5: e11431.

4 Thyagarajan B, Smith LJ, Barr RG, et al. Association of circulating adhesion molecules with lung function. The CARDIA study. Chest 2009; 135: 1481-1487.

5 Aaron CP, Schwartz JE, Bielinski SJ, et al. Intercellular adhesion molecule 1 and progression of percent emphysema: the MESA Lung Study. Respir Med 2015; 109: 255-264.

6 Hughes GH, Cutter G, Donahue R, et al. Recruitment in the Coronary Artery Disease Risk Development in Young Adults (CARDIA) Study. Control Clin Trials 1987; 8: Suppl., 68S-73S.

$7 \quad$ Kalhan R, Dransfield MT, Colangelo LA, et al. Respiratory Symptoms in Young Adults and Future Lung Disease. The CARDIA Lung Study. Am J Respir Crit Care Med 2018; 197: 1616-1624.

8 Zemans RL, Jacobson S, Keene J, et al. Multiple biomarkers predict disease severity, progression and mortality in COPD. Respir Res 2017; 18: 117.

9 Ostridge K, Williams NP, Kim V, et al. Relationship of CT-quantified emphysema, small airways disease and bronchial wall dimensions with physiological, inflammatory and infective measures in COPD. Respir Res 2018; 19: 31.

10 Newton AH, Danahy DB, Chan MA, et al. Timely blockade of ICAM-1.LFA-1 interaction prevents disease onset in a mouse model of emphysema. Immunotherapy 2015; 7: 621-629. 